\title{
Photo- and electro-excitation of the $\Delta$-resonance at MAMI
}

\author{
H. Schmieden ${ }^{\mathrm{a}}$ \\ Physikalisches Institut, Universität Bonn, Germany \\ / \\ Published online: 15 May 2006 - C Società Italiana di Fisica / Springer-Verlag 2006
}

\begin{abstract}
Over the last decade accurate experiments at MAMI played an essential role to improve our understanding of the nucleon to $\Delta(1232)$ transition. Originally to a large extent motivated through intraquark hyperfine interactions anticipated in QCD-inspired quark models they showed that pionic degrees of freedom are essential. The meson cloud is mainly responsible for the observed quadrupole excitation strength and affects the magnetic dipole transition strength as well.
\end{abstract}

PACS. 13.60.Le Meson production - 13.40.-f Electromagnetic processes and properties - 14.20.Gk Baryon resonances with $S=0$

\section{Introduction}

At very high energy and momentum transfers in deep inelastic lepton scattering, proton and neutron reveal their substructure of pointlike, almost massless spin- $1 / 2$ constituents, the quarks, and of gluons as the exchange bosons mediating the color force between them. In this regime of asymptotic freedom Quantum Chromodynamics is well established as the basic underlying theory of strong interaction. However, at momentum transfers corresponding to the nucleons' size, the nonlinear strong couplings prohibit the solution of the QCD field equations using perturbation theory. Hence, basic properties such as mass, size and excitation spectrum are only qualitatively [1] understood and remain still a domain of models [2]. To prove that QCD provides also the correct theory at the confinment scale is the challenge of Lattice calculations, currently stepping beyond quenched approximations [3] by realistic lightquark vacuum polarisation and chiral quark actions $[4,5]$.

Analogously to atomic spectroscopy at the threshold to the era of quantum mechanics, baryon spectroscopy serves today as a tool to improve our understanding of the inner dynamics of the nucleon. Generally, the high level density of excited states in combination with their short lifetimes, and thus large natural widths, provides an annoying experimental obstacle. However, there are two states which can be experimentally almost exclusively prepared, outstanding in cross section and well separated in mass from their neighbours. The $N(1535) S_{11}$ negative-parity partner of the nucleon which selectively couples to the $\eta$-nucleon final state. Its mass puts it at the very edge of the energy range accessible with MAMI-B. Contrary, the decuplet ground state $\Delta(1232) P_{33}$ couples almost entirely into the

\footnotetext{
a e-mail: schmieden@physik.uni-bonn.de
}

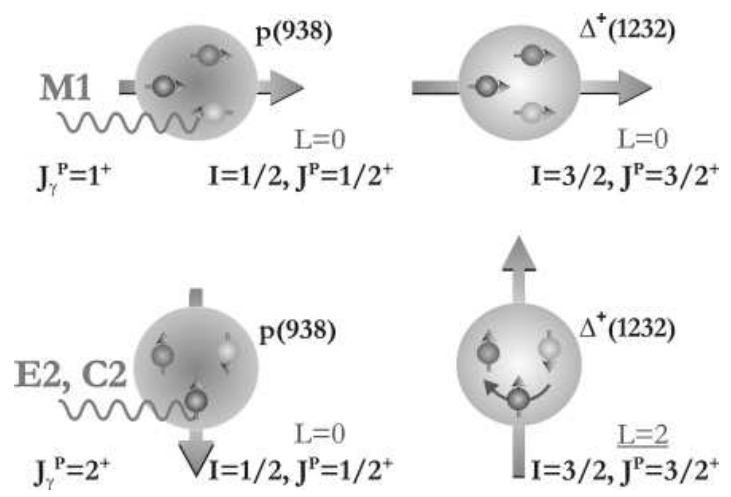

Fig. 1. Photoexcitation of the $N \rightarrow \Delta$ transition. Top: M1 photon generating a spin-flip transition; bottom: E2 or C2 photon introducing angular momentum $L=2$.

$\pi$-nucleon channel, perfectly fitting the present nominal MAMI energy and thus most suited for a detailed study.

From the viewpoint of the inner quark dynamics the nucleon to $\Delta(1232)$ transition is very interesting. In the $S U(6)$ symmetric constituent quark model it corresponds to a pure spin-flip of one of the quarks, yielding the spin $3 / 2$ of the $\Delta(1232)$. Electromagnetic excitation requires thus an M1 magnetic dipole photon as schematically depicted in fig. 1 (top). Parity and angular momentum conservation would alternatively allow the absorption of an $L=2$ photon, coupling together with the nucleons spin $1 / 2$ to $J=3 / 2$, cf. fig. 1 (bottom). However, this requires $L=2$ quadrupole components in one or both of the nucleons and deltas quark wave functions, in analogy to the deuteron in nuclear physics. As in the latter case, the quadrupole components can be associated with a spherical deformation of the system. They originate from 
tensor parts in the interaction of the constituents which, in QCD-motivated models, are attributed to the color hyperfine interaction among the quarks $[6,7]$.

While most of our knowledge about the nucleon excitation spectrum stems from pion-nucleon scattering experiments, those are insensitive to the quadrupole strength in the nucleon to $\Delta(1232)$ transition. Due to the positive parity of both nucleon and $\Delta$, the angular momentum $\Delta L=1$ magnetic transition is generated in $\pi$ - $N$ scattering, but not the $\Delta L=2$ one. The latter requires photons which provide positive parity with both $\Delta L=1$ and $\Delta L=2$.

For photon energies up to $2000 \mathrm{MeV}$, fig. 2 shows the total absorption cross section of real photons on the proton. The $\Delta(1232)$ resonance exhibits almost isolated around $E_{\gamma}=340 \mathrm{MeV}$, whereas the second and third resonance regions are composed of numerous overlapping resonances which, in the total absorption cross section, cannot be separated. Due to their short life time, the direct detection of the resonances is precluded. Since the $\Delta(1232)$ almost exclusively decays into pion and nucleon, pion photo- and electro-production off the nucleon provide the major experimental tools for the investigation of its properties.

\section{Kinematics and cross section of pion photo- and electro-production}

The kinematics of pion electroproduction are shown in fig. 3 at the example of the $e+p \rightarrow e+p+\pi^{0}$ reaction. The electron is scattered by the laboratory angle $\vartheta_{e}$ and the exchanged virtual photon transfers the difference between incoming and scattered electron energies and momenta, $\omega=E-E^{\prime}$ and $\boldsymbol{q}=\boldsymbol{k}-\boldsymbol{k}^{\prime}$, respectively. $Q^{2}=-q_{\mu} q^{\mu}>0$ represents the invariant mass of the virtual photon. The electron scattering plane is spanned by the unit vectors $\hat{\boldsymbol{z}}=\hat{\boldsymbol{q}}=\boldsymbol{q} /|\boldsymbol{q}|, \hat{\boldsymbol{y}}=\hat{\boldsymbol{k}} \times \hat{\boldsymbol{k}}^{\prime}$, and $\hat{\boldsymbol{x}}=\hat{\boldsymbol{y}} \times \hat{\boldsymbol{z}}$.

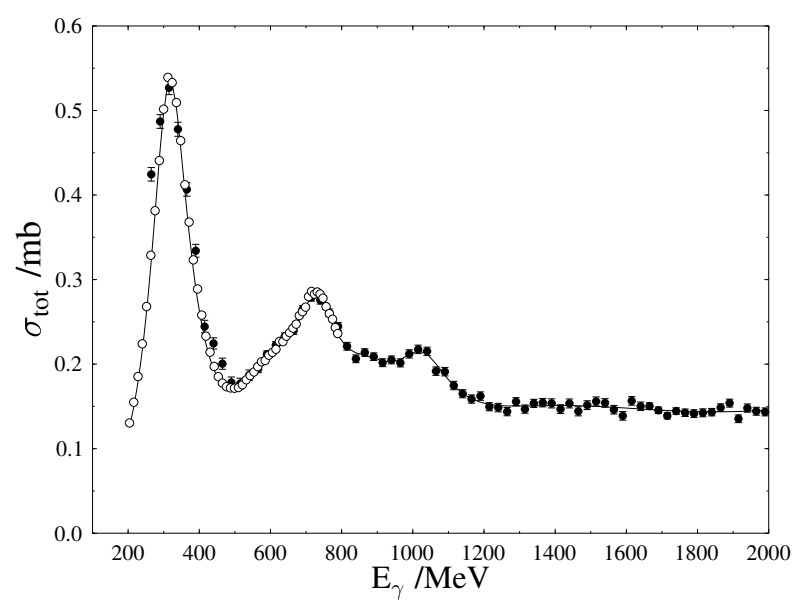

Fig. 2. World data of the real photon total absorption cross section as a function of the photon energy [8]. The open and full circles represent the data from MAMI [9] and NINA [10]. The parameterization after [11] is shown as the solid line.

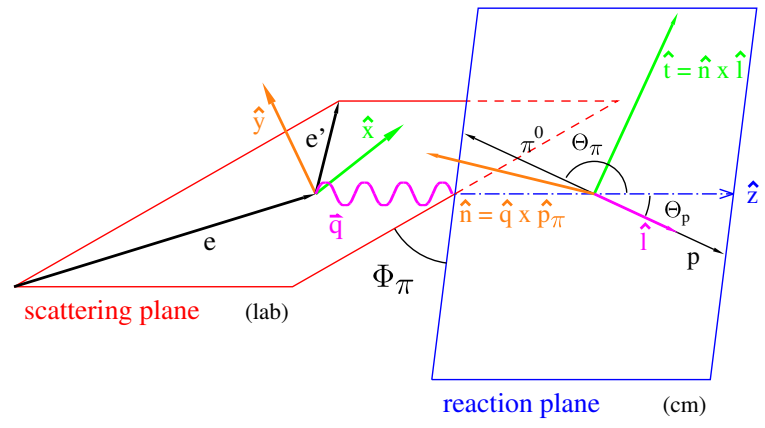

Fig. 3. Kinematics of pion electroproduction. The vectors of the reaction plane are in the rest frame of the $\Delta$.

In its rest frame, the recoiling hadronic system decays back to back into $\pi^{0}$ and proton. $\Theta_{\pi}$ and $\Theta_{p}$ denote the pion and proton angles with respect to $\boldsymbol{q}$ in the hadronic cm frame. The reaction plane, which is given by $\hat{\boldsymbol{l}}=\hat{\boldsymbol{p}}_{p}^{\mathrm{cm}}$, $\hat{\boldsymbol{n}}=\hat{\boldsymbol{q}} \times \hat{\boldsymbol{p}}_{\pi}^{\mathrm{cm}}$, and $\hat{\boldsymbol{t}}=\hat{\boldsymbol{n}} \times \hat{\boldsymbol{l}}$, is tilted against the electron scattering plane by the angle $\Phi$.

In one photon exchange approximation the fivefold differential cross section

$$
\frac{\mathrm{d}^{5} \sigma}{\mathrm{d} E_{e} \mathrm{~d} \Omega_{e} \mathrm{~d} \Omega_{\pi}^{\mathrm{cm}}}=\Gamma \frac{\mathrm{d}^{2} \sigma_{v}}{\mathrm{~d} \Omega_{\pi}^{\mathrm{cm}}}
$$

factorizes into the virtual photon flux,

$$
\Gamma=\frac{\alpha}{2 \pi^{2}} \frac{E^{\prime}}{E} \frac{k_{\gamma}}{Q^{2}} \frac{1}{1-\epsilon},
$$

and $\mathrm{d}^{2} \sigma_{v} / \mathrm{d} \Omega_{\pi}^{\mathrm{cm}}$, the virtual photon cm cross section. $\alpha$ denotes the fine structure constant, $k_{\gamma}=\left(W^{2}-m_{p}^{2}\right) / 2 m_{p}$ the real photon equivalent laboratory energy for the excitation of the target with mass $m_{p}$ to the cm energy $W$, and $\epsilon=\left[1+\left(2|\boldsymbol{q}|^{2} / Q^{2}\right) \tan ^{2} \frac{\vartheta_{e}}{2}\right]^{-1}$ the photon polarisation parameter.

Without target or recoil polarisation, the virtual photon cross section is given by [12]

$$
\begin{aligned}
\frac{\mathrm{d}^{2} \sigma_{v}}{\mathrm{~d} \Omega_{\pi^{c m}}}= & \lambda \cdot\left[R_{T}+\epsilon_{L} R_{L}+c_{+} R_{L T} \cos \Phi+\right. \\
& \left.\epsilon R_{T T} \cos 2 \Phi+P_{e} c_{-} R_{L T^{\prime}} \sin \Phi\right] .
\end{aligned}
$$

The ratio $\lambda=\left|\boldsymbol{p}_{\pi}^{\mathrm{cm}}\right| / k_{\gamma}^{\mathrm{cm}}$ is determined by the pion $\mathrm{cm}$ momentum $\boldsymbol{p}_{\pi}^{\mathrm{cm}}$ and $k_{\gamma}^{\mathrm{cm}}=\left(m_{p} / W\right) k_{\gamma}$. The structure functions, $R_{i}$, parameterize the response of the hadronic system to the various polarisation states of the photon field, which are described by the transverse and longitudinal polarisation, $\epsilon$ and $\epsilon_{L}=\frac{Q^{2}}{\omega_{\mathrm{cm}}^{2}} \epsilon$, also contained within the factors $c_{ \pm}=\sqrt{2 \epsilon_{L}(1 \pm \epsilon)}$. The degree of longitudinal electron polarisation is denoted by $P_{e}$.

In lowest order, the tree graphs depicted in fig. 4 contribute to the cross section in the $\Delta(1232)$ resonance region. For $\pi^{0}$ production the pion pole and the contact term vanish, because the photon cannot couple to the chargeless pion. Thus, usually the $\pi^{0}$ channel is chosen to tag the $\Delta(1232)$ intermediate state. Nevertheless, the unambiguous tagging is hampered by the inevitable background 

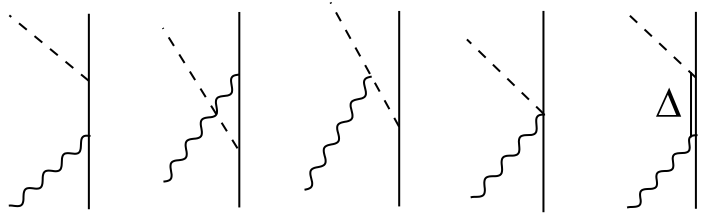

Fig. 4. Lowest-order graphs of single-pion electroproduction.

from non-resonant $\pi$ production (cf. fig. 4). Therefore, to extract the small quadrupole admixture, a full multipole analysis would be desirable in analogy to $\pi$ - $N$ scattering. However, in pion production experiments with real and, in particular, virtual photons this is much more difficult to achieve, because more invariant amplitudes need to be independently determined.

\subsection{Multipole decomposition}

The response functions $R$ of eq. (3) can be decomposed into pion multipoles of the final state. In $S$ and $P$ wave approximation this yields $[12,13]$ :

$$
\begin{aligned}
& R_{L}=\tilde{\lambda}^{2}\left[\left|S_{0+}\right|^{2}+4\left|S_{1+}\right|^{2}+\left|S_{1-}\right|^{2}\right. \\
& -4 \Re e\left\{S_{1+}^{*} S_{1-}\right\}+2 \cos \Theta \Re e\left\{S_{0+}^{*}\left(4 S_{1+}+S_{1-}\right)\right\} \\
& \left.+12 \cos ^{2} \Theta\left(\left|S_{1+}\right|^{2}+\Re e\left\{S_{1+}^{*} S_{1-}\right\}\right)\right] \text {, } \\
& R_{T}=\left|E_{0+}\right|^{2}+\frac{1}{2}\left|2 M_{1+}+M_{1-}\right|^{2} \\
& +\frac{1}{2}\left|3 E_{1+}-M_{1+}+M_{1-}\right|^{2} \\
& +2 \cos \Theta \Re e\left\{E_{0+}^{*}\left(3 E_{1+}+M_{1+}-M_{1-}\right)\right\} \\
& +\cos ^{2} \Theta\left[\left|3 E_{1+}+M_{1+}-M_{1-}\right|^{2}\right. \\
& -\frac{1}{2}\left|2 M_{1+}+M_{1-}\right|^{2} \\
& \left.\left.-\frac{1}{2}\left|3 E_{1+}-M_{1+}+M_{1-}\right|^{2}\right\}\right] \text {, } \\
& R_{T T}=3 \sin ^{2} \Theta \quad\left[\frac{3}{2}\left|E_{1+}\right|^{2}-\frac{1}{2}\left|M_{1+}\right|^{2}\right. \\
& \left.-\Re e\left\{E_{1+}^{*}\left(M_{1+}-M_{1-}\right)+M_{1+}^{*} M_{1-}\right\}\right], \\
& R_{L T}=-\sin \Theta \tilde{\lambda} \Re e\left\{S_{0+}^{*}\left(3 E_{1+}-M_{1+}+M_{1-}\right)\right. \\
& -\left(2 S_{1+}^{*}-S_{1-}^{*}\right) E_{0+} \\
& +6 \cos \Theta\left(S_{1+}^{*}\left(E_{1+}-M_{1+}+M_{1-}\right)\right. \\
& \left.\left.+S_{1-}^{*} E_{1+}\right)\right\}, \\
& R_{L T^{\prime}}=\sin \Theta \tilde{\lambda} \Im m\left\{S_{0+}^{*}\left(3 E_{1+}-M_{1+}+M_{1-}\right)\right. \\
& -\left(2 S_{1+}^{*}-S_{1-}^{*}\right) E_{0+} \\
& +6 \cos \Theta\left(S_{1+}^{*}\left(E_{1+}-M_{1+}+M_{1-}\right)\right. \\
& \left.\left.+S_{1-}^{*} E_{1+}\right)\right\} \text {. }
\end{aligned}
$$

\begin{tabular}{|c|c|c|c|c|c|}
\hline \multicolumn{2}{|c|}{$\gamma-N$ system } & \multicolumn{3}{|c|}{$\pi-N$ system } & \multirow{2}{*}{ parity } \\
\hline$L_{\gamma}$ & $\gamma$-multipole & $J$ & $l_{\pi}$ & $\pi$-multipole & \\
\hline 0 & $C 0$ & $1 / 2$ & 1 & $S_{1-}$ & + \\
\hline \multirow[b]{2}{*}{1} & $E 1 / C 1$ & $\begin{array}{l}1 / 2 \\
3 / 2\end{array}$ & $\begin{array}{l}0 \\
2\end{array}$ & $\begin{array}{l}E_{0+} / S_{0+} \\
E_{2-} / S_{2-}\end{array}$ & - \\
\hline & $M 1$ & $\begin{array}{l}1 / 2 \\
3 / 2\end{array}$ & $\begin{array}{l}1 \\
1\end{array}$ & $\begin{array}{l}M_{1-} \\
M_{1+}\end{array}$ & + \\
\hline \multirow[b]{2}{*}{2} & $E 2 / C 2$ & $\begin{array}{l}3 / 2 \\
5 / 2\end{array}$ & $\begin{array}{l}1 \\
3\end{array}$ & $\begin{array}{l}E_{1+} / S_{1+} \\
E_{3-} / S_{3-}\end{array}$ & + \\
\hline & $M 2$ & $\begin{array}{l}3 / 2 \\
5 / 2\end{array}$ & $\begin{array}{l}2 \\
2\end{array}$ & $\begin{array}{l}M_{2-} \\
M_{2+}\end{array}$ & - \\
\hline
\end{tabular}

$\tilde{\lambda}=\omega_{\mathrm{cm}} /\left|\boldsymbol{q}_{\mathrm{cm}}\right|$ is a current conservation factor related to the definition of the amplitudes. The photon and pion multipoles up to order $L_{\gamma}=2$ are summarized in table 1. According to their parity the photon multipoles are classified
Table 1. Photon and pion multipoles up to $L_{\gamma}=2$.

as electric/coulombic $(E / C)$ or magnetic $(M)$. The pion multipoles $A_{l_{\pi} \pm}^{I}$ are characterized through their magnetic, electric or scalar (longitudinal) nature, $A=M, E, S(L)$, the isospin, $I$, and the pion-nucleon relative angular momentum, $l_{\pi}$. The coupling of $l_{\pi}$ with the nucleon spin to the total angular momentum, $J$, is indicated by \pm . Omission of the isospin index as in eqs. (4-8) indicates amplitudes in the $p \pi^{0}$ charge channel throughout this paper.

In general, the unambiguous tagging of a resonance requires the multipoles to be determined in the appropriate isospin channel. However, at the resonance position of the $\Delta(1232)$ the $p \pi^{0}$ amplitudes provide a very good approximation to the separated isospin components. Both methods, simultaneous measurement of $\pi^{0}$ and $\pi^{+}$production and sole $\pi^{0}$ production, have been exploited.

\section{Experimental methods and results}

The sensitivity to the $N \rightarrow \Delta(1232)$ quadrupole amplitudes is directly illustrated by the $S$ and $P$ wave multipole decomposition of the cross section in eqs. (4-8). All structure functions contain $E_{1+}$ or $S_{1+}$ amplitudes, either quadratically or as bilinear combinations.

The smallness of the quadrupole amplitudes makes a determination of their square against the leading $\left|M_{1+}\right|^{2}$ very difficult, e.g., a Rosenbluth separation of $R_{L}$ and $R_{T}$ for the extraction of $\left|S_{1+}\right|^{2}$. It is much more promising to exploit interferences with the dominating $M_{1+}$ multipole. Thus, the experiments based on unpolarised electron beams extracted the quadrupole over dipole ratios

$$
\operatorname{EMR}^{\pi^{0}}=\frac{\Re e\left\{E_{1+}^{*} M_{1+}\right\}}{\left|M_{1+}\right|^{2}}
$$

and

$$
\mathrm{CMR}^{\pi^{0}}=\frac{\Re e\left\{S_{1+}^{*} M_{1+}\right\}}{\left|M_{1+}\right|^{2}}
$$

from measurements of the $R_{T T}$ and $R_{L T}$ type structure functions. 


\subsection{Real photon experiments}

With real photon beams, i.e. $Q^{2}=0$, the longitudinal parts of the cross section vanish. Of eqs. (4-8) thus only $R_{T}$ and $R_{T T}$ contribute and the cross section is often written in the form [12]

$$
\frac{\mathrm{d}^{2} \sigma}{\mathrm{d} \Omega_{\pi}^{c m}}=\left[\frac{\mathrm{d}^{2} \sigma}{\mathrm{d} \Omega_{\pi}^{c m}}\right]_{0} \cdot\left[1-P_{\gamma} \Sigma \cos 2 \Phi\right],
$$

where $\left[\frac{\mathrm{d}^{2} \sigma}{\mathrm{d} \Omega_{\pi}^{c m}}\right]_{0}$ denotes the unpolarised cross section and $P_{\gamma}$ is the degree of linear polarisation of the photon beam. The photon-beam asymmetry $\Sigma$ represents the ratio $R_{T T} / R_{T}$ and thus enters the cross section with the $\cos 2 \Phi$ modulation characteristic for the directional sensitivity against the plane of linear polarisation. This plane is intrinsically fixed by kinematic constraints in electron scattering, usually the detection of the final-state electron under a certain angle. Contrary, real photon beams from electron bremsstrahlung are a homogeneous superposition of all polarisation directions. The net polarisation consequently vanishes.

To obtain linear polarised photon beams it is necessary to fix the electron scattering plane in the bremsstrahlung process. This can be achieved by off-axis tagging or, as exploited at MAMI, through coherent bremsstrahlung off a diamond crystal. Under certain kinematic conditions depending on the crystal alignment relative to the electron beam the crystal lattice, similar to the Mössbauer effect, coherently takes the bremsstrahlung recoil instead of individual nuclei. This generates intensity peaks in the otherwise $\simeq 1 / E_{\gamma}$ distribution of the bremsstrahlung spectrum. Within the energy region of the coherent peak the crystal orientation determines a particular electron scattering plane. Hence, the photon beam is linearly polarised and the degree of linear polarisation is related to the intensity excess over the incoherent spectrum.

According to eq. (11), linear polarised beams with $P_{\gamma} \neq 0$ enable the contribution of the beam asymmetry $\Sigma$ to the observed cross section. Its size is determined by interferences of the type $\left|M_{1+}\right|^{2}+6 \Re e\left\{E_{1+}^{*} M_{1+}\right\}+\cdots$

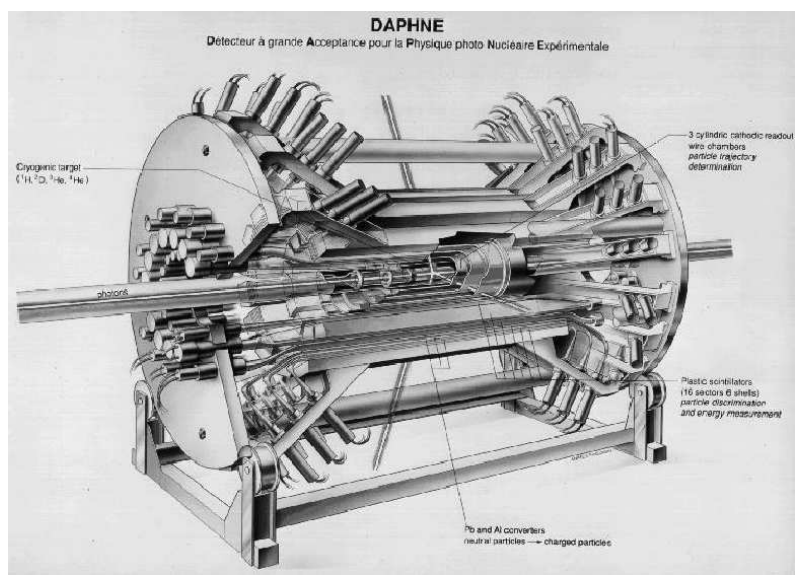

Fig. 5. The cylinder-symmetric DAPHNE detector (Figure courtesy of MAMI-A2 collaboration). which provide a very high sensitivity to the EMR. In order to exploit those experimentally, the $\cos 2 \Phi$ azimuthal modulation of the cross section needs to be determined. A detector with cylinder symmetry is ideally suited for this purpose. The first experiments of the A2 collaboration at MAMI consequently used the DAPHNE setup to detect the recoil protons from the $\gamma+p \rightarrow \pi^{0}+p$ reaction, schematically depicted in fig. $5[14,15]$.

Results of such measurements are shown in fig. 6. Exploiting the cleanly measured azimuthal modulation (left part of figure) the photon beam asymmetries (right part) can be determined as a function of polar angle over the entire energy region of the $\Delta(1232)$ resonance. In conjunction with the simultaneously measured differential cross sections it is possible to perform a detailed mulipole analysis. Results are depicted in fig. $7[15,16]$. Furthermore, including the results of the reaction $\gamma+p \rightarrow \pi^{+}+n$ with linearly polarised photon beam enables an isospin decomposition. At the resonance position it provides EMR results in agreement with the $\pi^{0}$ channel alone. As final result [15] the electric quadrupole to magnetic dipole ratio
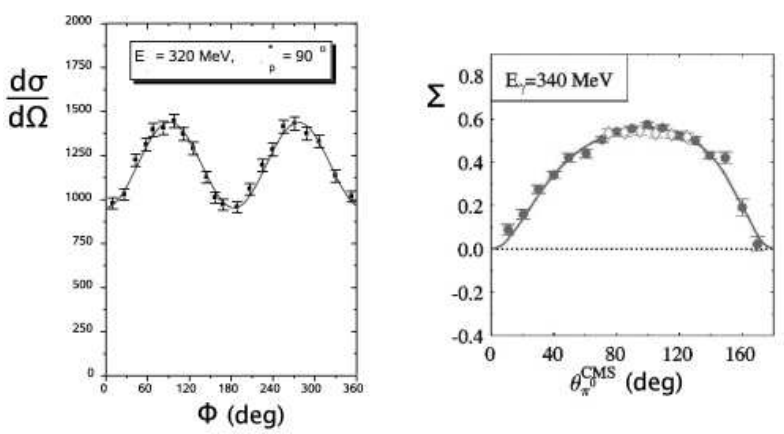

Fig. 6. Left: Measured relative cross section of the reaction $\gamma+p \rightarrow \pi^{0}+p$ using linearly polarised photon beam as a function of the azimuthal angle $\Phi$ at fixed polar angle $\Theta_{\pi}^{c m}=$ $90^{\circ}$. Right: Polar-angle dependence of the extracted photon beam asymmetry.

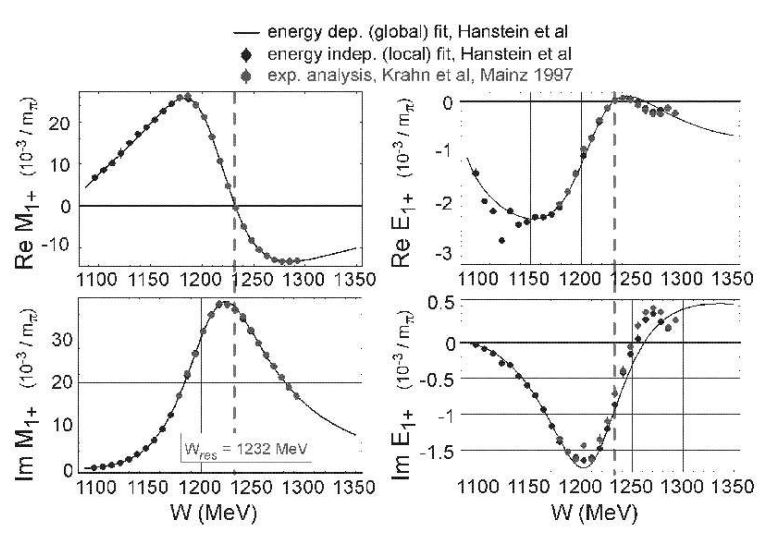

Fig. 7. Multipole fits based on measurements of cross section and photon asymmetry using linearly polarised real photon beams [16]. 


$$
\frac{\Re e\left\{E_{1+}^{*} M_{1+}\right\}}{\left|M_{1+}\right|^{2}}=\left(-2.5 \pm 0.1_{\text {stat }} \pm 0.2_{\text {syst }}\right) \%
$$

was obtained.

The beam asymmetry had also been extracted using the large acceptance TAPS photon detector, covering the full polar angular range for the $\pi^{0}$ decay photons [17]. Despite the coplanar detector arrangement, $\Sigma$ could be determined through the deliberate rotation of the photon polarisation plane.

\subsection{Virtual photon experiments}

Due to the nonzero mass of the exchange photon, in electroproduction also longitudinal pieces contribute, in total four structure functions with unpolarised electron beam and a fifth one with longitudinally polarised beam. If either a polarised target is provided in the initial state or the final state proton polarisation is determined, then 13 more structure functions enter the cross section, i.e. a total of 18 . Not all of those are independent.

In addition to the EMR now also the CMR ( $c f$. eq. (10)) is accessible. As in the photoproduction case, sufficient sensitivity is only obtained through interferences of the $S_{1+}$ amplitude with the dominating $M_{1+}$.

\subsubsection{Unpolarised electroproduction}

According to the discussion of sect. (3.1), in electron scattering a linearly polarised photon field is already obtained without the need to polarise the electrons. Thus, the EMR can be determined similar to real photon experiments with a large acceptance detector $[18,19]$. The kinematic focussing at large $Q^{2}$ enables full centre-of-mass coverage using a relatively small acceptance in the laboratory, e.g. of magnetic spectrometers [20]. At smaller $Q^{2}$ the required angular range must be covered by subsequent settings of the spectrometers. This technique has been used for the MAMI experiments in order to determine the $\mathrm{CMR}^{\pi^{0}}$ from the longitudinal-transverse interference part of the cross section. Since $R_{L T}$ comes along with a $\cos \Phi$ azimuthal modulation, $c f$. eq. (3), it is sufficient to measure the recoil protons of the $p\left(e, e^{\prime} p\right) \pi^{0}$ reaction within the electron scattering plane, i.e. within the laboratory floor plane. At each $\pi^{0}$ centre-of-mass polar angle $\theta_{\pi^{0}}^{c m}$, the cosine term exhibits through a cross section asymmetry in measurements left $\left(\Phi=0^{\circ}\right)$ and $\operatorname{right}\left(\Phi=180^{\circ}\right)$ of the direction of three-momentum transfer, $\boldsymbol{q}$ :

$$
\rho_{L T}\left(\theta_{\pi^{0}}^{c m}\right):=\frac{\mathrm{d} \sigma_{v}\left(\Phi=0^{\circ}\right)-\mathrm{d} \sigma_{v}\left(\Phi=180^{\circ}\right)}{\mathrm{d} \sigma_{v}\left(\Phi=0^{\circ}\right)+\mathrm{d} \sigma_{v}\left(\Phi=180^{\circ}\right)} .
$$

According to eq. (3), it is related to the partial responses via

$$
\rho_{L T}\left(\theta_{\pi^{0}}^{c m}\right)=\frac{c_{+} R_{L T}}{R_{T}+\epsilon_{L} R_{L}+\epsilon R_{T T}} .
$$

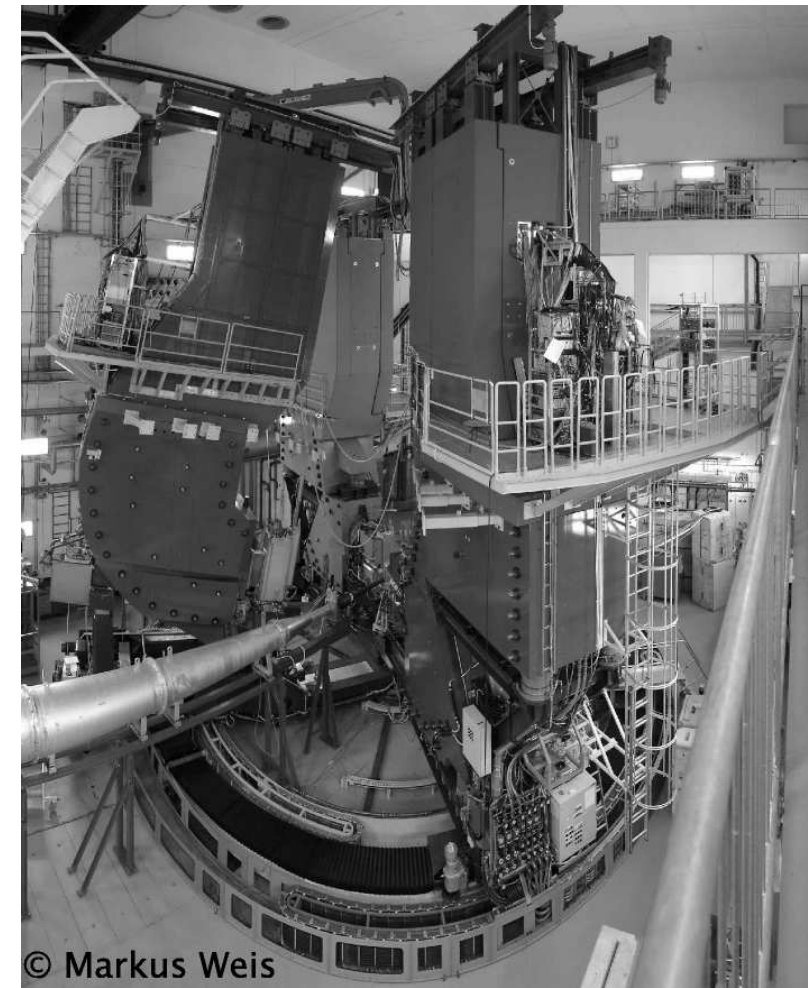

Fig. 8. Three-Spectrometer-Setup of the MAMI-A1 collaboration. Spectrometer B (left) is lifted out of plane by 10 degrees (see text). Picture courtesy of Markus Weis.

The sensitivity to $S_{1+}$ can be seen from the decomposition of eq. (14) into the leading $S$ and $P$ partial waves. At the $\Delta(1232)$-resonance position the asymmetry

$$
\rho_{L T}\left(\theta_{\pi^{0}}^{c m}\right) \simeq f\left(\theta_{\pi^{0}}^{c m}\right) \frac{\Re\left\{\left(S_{0+}^{*}+6 S_{1+}^{*} \cos \theta_{\pi^{0}}^{c m}\right) M_{1+}\right\}}{\left|M_{1+}\right|^{2}}
$$

is approximated. Thus measurements of $\rho_{L T}$ in the forward $\left(\theta_{1}\right)$ and backward cm-hemisphere $\left(\theta_{2}=\pi-\theta_{1}\right)$ allow the extraction of $S_{1+} / M_{1+}$ and, simultaneously, of $S_{0+} / M_{1+}$, i.e. the main contribution of inherent nonresonant background:

$$
\begin{aligned}
& \frac{\Re\left\{S_{1+}^{*} M_{1+}\right\}}{\left|M_{1+}\right|^{2}}=f_{1}\left(\theta_{1,2}\right) \cdot\left[\rho_{L T}\left(\theta_{1}\right)-\rho_{L T}\left(\theta_{2}\right)\right]+C_{1}, \\
& \frac{\Re\left\{S_{0+}^{*} M_{1+}\right\}}{\left|M_{1+}\right|^{2}}=f_{0}\left(\theta_{1,2}\right) \cdot\left[\rho_{L T}\left(\theta_{1}\right)+\rho_{L T}\left(\theta_{2}\right)\right]+C_{0} .
\end{aligned}
$$

The functions $f$ denote kinematic factors, $C_{0}$ and $C_{1}$ contributions of multipoles beyond the simple approximation.

Using the 3-Spectrometer-Setup of the MAMI-A1 collaboration shown in fig. 8, the asymmetries

$$
\begin{aligned}
\rho_{L T}\left(\theta_{\pi^{0}}^{c m}=160^{\circ}\right) & =\left(12.18 \pm 0.27_{\text {stat }} \pm 0.82_{\text {sys }}\right) \%, \\
\rho_{L T}\left(\theta_{\pi^{0}}^{c m}=20^{\circ}\right) & =\left(-11.68 \pm 2.36_{\text {stat }} \pm 2.36_{\text {sys }}\right) \%
\end{aligned}
$$

have been measured [21]. They are shown in fig. 9 along with different calculations. The prediction of Sato and Lee [22] describes the asymmetries quite well, to a lesser 


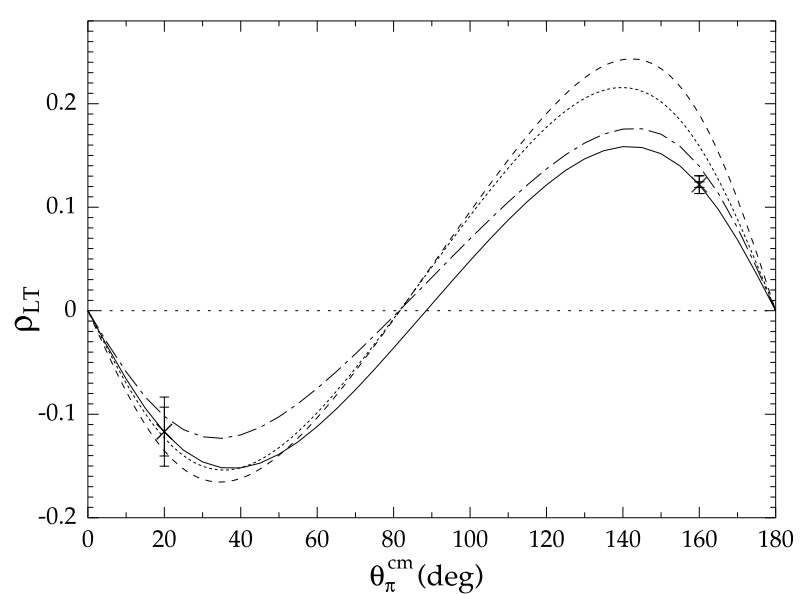

Fig. 9. Left-right asymmetries $\rho_{L T}$ measured in $\pi^{0}$ production with unpolarised electron beam compared to model predictions from MAID2003 [23] (dotted), DMT2001 [24,25] (dashed), Sato/Lee [22] (dashed dotted). The full curve represents a MAID2003 re-fit [21]. The inner and outer errors are purely statistical and quadratically summed statistical and systematical, respectively.

extent the calculation within the dynamical model of Kamalov and Yang [25]. Also the standard MAID2003 parametrisation [23] provided only moderate agreement, in particular at backward pion angle. This was resolved through a re-fit including also polarisation data as described in the following subsect. 3.2.2.

Using the MAID re-fit, the multipole ratios of eqs. (16) and (17) were determined to

$$
\begin{aligned}
& \frac{\Re\left\{S_{1+}^{*} M_{1+}\right\}}{\left|M_{1+}\right|^{2}}=(-5.45 \pm 0.42) \%, \\
& \frac{\Re\left\{S_{0+}^{*} M_{1+}\right\}}{\left|M_{1+}\right|^{2}}=(2.56 \pm 2.25) \% .
\end{aligned}
$$

The $S_{0+}$ result is compared to older measurements in fig. 10. It practically excludes that a large negative $\mathrm{CMR}^{\pi^{0}}$ found by ref. [26] at $Q^{2}=0.12(\mathrm{GeV} / c)^{2}$ can be explained by the particular contribution of a large negative $S_{0+} / M_{1+} \simeq-10 \%$ [27] in forward pion kinematics.

\subsubsection{Polarised electron beams: 5 th structure function}

$R_{L T^{\prime}}$, the 5 th structure function of eq. (3), measures the imaginary part of the same interference which $R_{L T}$ and $\rho_{L T}$ contain the real part of. This is obvious from comparison of the $S$ - and $P$-wave decompositions in eqs. (7) and (8). Experimentally, $R_{L T^{\prime}}$ can be extracted through the cross section asymmetry with regard to the flip of beam helicity between \pm 1 :

$$
\begin{aligned}
& \rho_{L T^{\prime}}=\frac{\mathrm{d} \sigma^{+}-\mathrm{d} \sigma^{-}}{\mathrm{d} \sigma^{+}-\mathrm{d} \sigma^{-}} \\
& =\frac{c_{-} R_{L T^{\prime}} \sin \Phi}{R_{T}+\epsilon_{L} R_{L}+c_{+} R_{L T} \cos \Phi+\epsilon R_{T T} \cos 2 \Phi} .
\end{aligned}
$$

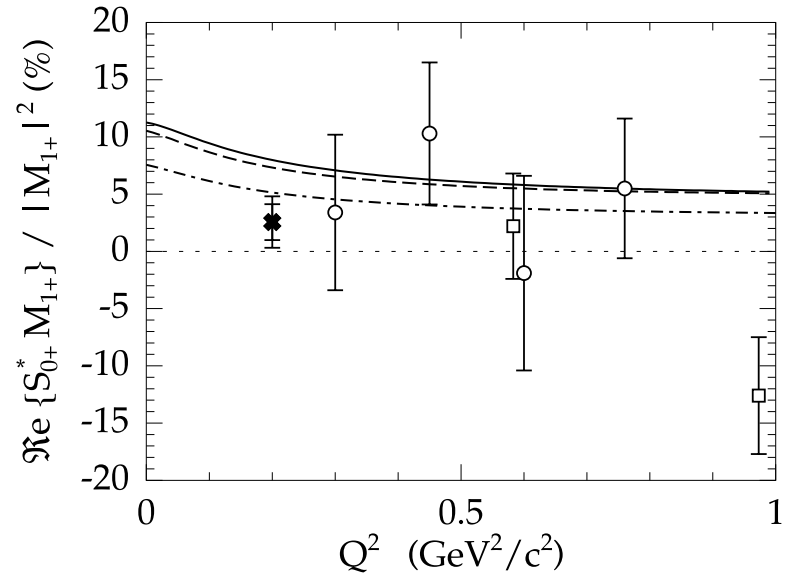

Fig. 10. Result for the $S_{0+}$ strength from $\pi^{0}$ electroproduction at low $Q^{2}$ (black cross). The open sympols represent older measurements from DESY [28] and NINA [29].

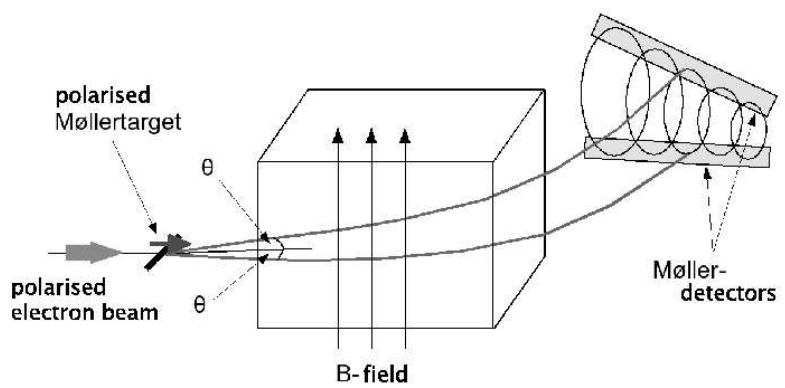

Fig. 11. Schematics of the Møller polarimeter of the MAMIA1 collaboration.

The measurement requires longitudinally polarised electron beam and, since $R_{L T^{\prime}}$ enters the cross section with $\sin \Phi$ (cf. eq. (3)), out-of-plane detection of the recoil protons or the scattered electrons of the $p\left(\boldsymbol{e}, e^{\prime} p\right) \pi^{0}$ reaction.

Intense and highly polarised electron beams are routinely available at MAMI [30]. The degree of longitudinal polarisation at the beam axis in the spectrometer hall is accurately measured by a Møller polarimeter, where the beam electrons are scattered off polarised electrons. Those are provided in an iron foil magnetised to saturation within the 4 Tesla field of a superconducting solenoid. Symmetric scattering kinematics is selected by momentum-specific detection of the outgoing electron pair behind a magnetic dipole field. The setup is schematically shown in fig. 11. A cm angular acceptance of $\delta \Theta / \Theta \simeq 20 \%$ around $\Theta_{\mathrm{cm}}=90^{\circ}$ makes the influence of the Fermi motion of the bound electrons, the so-called Levchuk effect [31], negligible [32].

Out-of-plane detection capability is provided by spectrometer B, which can be tilted up to $10^{\circ}$ [33] as visible in fig. 8. Using this setup the beam-helicity asymmetry was determined at the energy of the $\Delta(1232)$ resonance and $Q^{2}=0.2(\mathrm{GeV} / c)^{2}[34]$. The result is shown in fig. 12 .

The calculations within the dynamical models of Sato and Lee [22] as well as of DMT2001 [25], and the MAID2000 parametrisation [23] all were found to disagree 


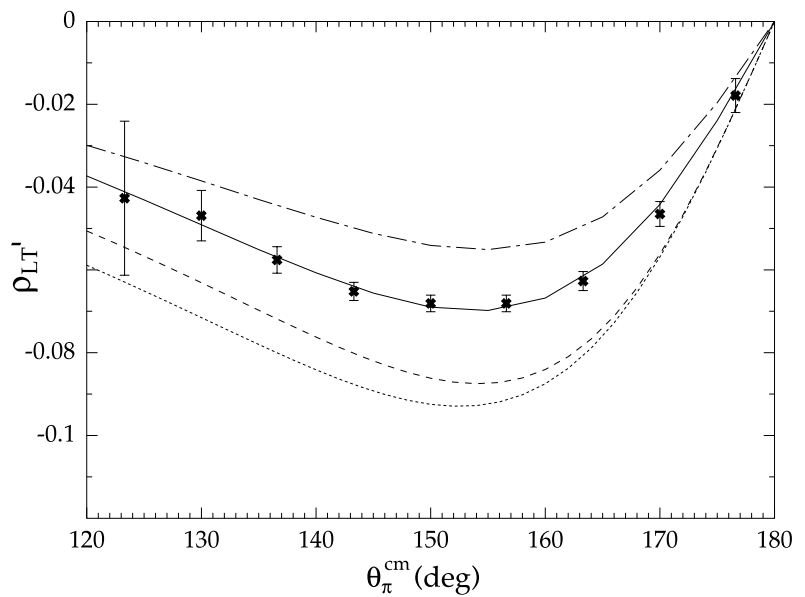

Fig. 12. Beam-helicity asymmetry $\rho_{L T^{\prime}}$ measured in $\pi^{0}$ production with longitudinally polarised electron beam. The dotted curve represents the original MAID2003 calculation [23]. The dashed-dotted and dashed curves are the results of the dynamical models of Sato-Lee [22] and Kamalov-Yang [25], respectively. The full curve is the MAID re-fit of ref. [21]. Errors are purely statistical.

with this measurement. However, rescaled by a factor 0.75 MAID described the asymmetry very well. The later refit of real and imaginary parts of the $S_{1+}$ and $S_{0+} \pi^{0}$ amplitudes in MAID2003 on basis of the MAMI $\rho_{L T}$ and $\rho_{L T^{\prime}}$ data provided a very satisfactory description of the polarisation data, $c f$. the full curve in fig. 12 , and of the unpolarised measurement discussed in the preceding section as well [21]. This underlines the absolute need to understand the physical background amplitudes before the small quadrupole contributions can be reliably extracted. Ideally, a complete experiment with respect to a multipole decompostion is required.

\subsubsection{Double Polarisation Experiments: Recoil proton polarimetry}

A complete experiment in the above sense would require to measure more than 11 independent observables over the energy range of the $\Delta(1232)$ and the full angular range. Even with application of Watson's theorem [35,36], which below $2 \pi^{0}$ threshold relates real and imaginary parts of the amplitudes, this presently is out of reach in pion electroproduction. In order to get as close as possible and thus minimise the model dependence in the extraction of the quadrupole amplitudes, it is mandatory to measure double polarisation observables.

In parallel kinematics of the $p\left(\boldsymbol{e}, e^{\prime} \boldsymbol{p}\right) \pi^{0}$ reaction ( $c f$. fig. 13) each of the three cartesian components of the proton polarisation, in addition to the unpolarised cross section $\sigma_{0}$, only depends on one specific structure func-

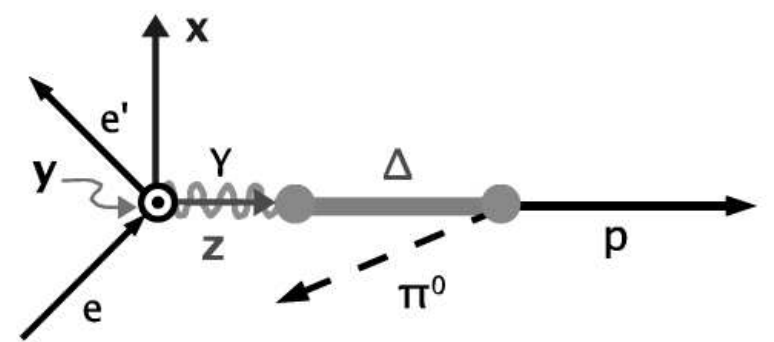

Fig. 13. Recoil polarisation components in parallel kinematics of the reaction $p\left(\boldsymbol{e}, e^{\prime} \boldsymbol{p}\right) \pi^{0}$. For visual clarity only, the $\pi^{0}$ is drawn slightly sideways. The $x, y$, and $z$ components are defined relative to the electron scattering plane.

tion [37]:

$$
\begin{aligned}
& \sigma_{0} P_{x}=\lambda \cdot P_{e} \cdot \sqrt{2 \epsilon_{L}(1-\epsilon)} R_{L T^{\prime}}^{t}, \\
& \sigma_{0} P_{y}=\lambda \cdot \sqrt{2 \epsilon_{L}(1+\epsilon)} R_{L T}^{n}, \\
& \sigma_{0} P_{z}=\lambda \cdot P_{e} \cdot \sqrt{1-\epsilon^{2}} R_{T T^{\prime}}^{l} .
\end{aligned}
$$

According to fig. 13 the axes are defined relative to the electron scattering plane and the virtual photon direction. This is the natural choice, since in parallel kinematics the recoil polarisation is completely determined by the angular momentum transfer from the photon field. Decomposition up to $S$ and $P$ partial waves,

$$
\begin{aligned}
\sigma_{0} P_{x}=P_{e} c_{-} \tilde{\lambda} \Re e\left\{\left(4 S_{1+}+S_{1-}-S_{0+}\right)^{*}\right. \\
\left.\left(M_{1+}-M_{1-}-E_{0+}+3 E_{1+}\right)\right\}, \\
\sigma_{0} P_{y}=-c_{+} \tilde{\lambda} \Im m\left\{\left(4 S_{1+}+S_{1-}-S_{0+}\right)^{*}\right. \\
\left.\left(M_{1+}-M_{1-}-E_{0+}+3 E_{1+}\right)\right\}, \\
\sigma_{0} P_{z}=P_{e} c_{0}\left[\left|M_{1+}\right|^{2}+\left|M_{1-}\right|^{2}+9\left|E_{1+}\right|^{2}+\left|E_{0+}\right|^{2}\right. \\
+\Re e\left\{6 E_{1+}^{*}\left(M_{1+}-M_{1-}\right)-2 M_{1+}^{*} M_{1-}\right. \\
\left.\left.-2 E_{0+}^{*}\left(M_{1+}-M_{1-}+3 E_{1+}\right)\right\}\right],
\end{aligned}
$$

reveals the high sensitivity to the quadrupole amplitudes. In particular, since $\sigma_{0}$ is dominated by $\left|M_{1+}\right|^{2}, P_{x}$ provides a rather direct measure of the $\mathrm{CMR}^{\pi^{0}}$. The factor $c_{0}=\sqrt{1-\epsilon^{2}}$ entering $P_{z}$ is related to the circular polarisation of the photon field.

Except the small contribution of $R_{L}$ to $\sigma_{0}$, in parallel kinematics $P_{z}$ is entirely determined by kinematic constants, i.e. the transfer of circular photon polarisation to the protons, since $R_{T T^{\prime}}^{l}=R_{T}$. The transverse components $P_{x}$ and $P_{y}$ measure real and imaginary parts of the same interferences, similar to $\rho_{L T}$ and $\rho_{L T^{\prime}}$ but in different combinations and with different weights of amplitudes. Thus, the model dependence in the extraction of the $\mathrm{CMR}^{\pi^{0}}$ is different from unpolarised measurements. Morever, also the composition of systematic errors is completely different due to the orthogonal experimental technique.

The first $N \rightarrow \Delta$ double-polarisation experiment ever has been performed at the 3-Spectrometer setup of MAMI [38]. Central to the experiment is the measurement of proton polarisation behind the focal plane of one of the spectrometers $[39,40]$. This is based on inclusive proton 
carbon scattering. Due to the strong spin-orbit coupling, the transverse polarisation components, $P_{n}$ and $P_{t}$, generate an azimuthal modulation of the unpolarized cross section, $\sigma_{0}^{C}$ :

$$
\sigma^{C}=\sigma_{0}^{C}\left(\Theta_{s}, T\right)\left[1+A_{C}\left(\Theta_{s}, T\right)\left(P_{t} \sin \Phi-P_{n} \cos \Phi\right)\right]
$$

The relative strength is determined by the known $[41,42]$ analyzing power, $A_{C}$.

The two polarisation components accessible at the focal plane must be traced back to the target through the spectrometer magnets. This complication on the one hand provides, on the other hand, the opportunity to determine the otherwise inaccessible longitudinal polarisation component. A separation of $P_{x}, P_{y}$ and $P_{z}$ is achieved since $x$ and $z$ components are odd under beam-helicity reversal, while $P_{y}$ is even (cf. eqs. (22)-(24)). At the energy of the $\Delta(1232)$ resonance and a momentum transfer of $Q^{2}=0.121(\mathrm{GeV} / c)^{2}$ the three polarisations were simultaneously measured to

$$
\begin{aligned}
P_{x} / P_{e} & =\left(-11.4 \pm 1.3_{\text {stat }} \pm 1.4_{\text {syst }}\right) \%, \\
P_{y} & =\left(-43.1 \pm 1.3_{\text {stat }} \pm 2.2_{\text {syst }}\right) \%, \\
P_{z} / P_{e} & =\left(56.2 \pm 1.5_{\text {stat }} \pm 2.6_{\text {syst }}\right) \% .
\end{aligned}
$$

Based on the $P_{x}$ result, the $\mathrm{CMR}^{\pi^{0}}$

$$
\frac{\Re\left\{S_{1+}^{*} M_{1+}\right\}}{\left|M_{1+}\right|^{2}}=\left(-6.4 \pm 0.7_{\text {stat }} \pm 0.8_{\text {syst }}\right) \%
$$

was extracted using the MAID parametrisation [38]. Within the errors this agrees with the alternative method of extraction using $P_{x} / P_{z}$, which experimentally provides the advantage that the magnitudes of both analysing power and beam polarisation drop out. Contrary, the ratio is affected by a larger uncertainty in spin precession of the $z$-component compared to $P_{x}$. To first order the latter would not precess at all in a homogeneous vertical-bend dipole field.

The result for the $\mathrm{CMR}^{\pi^{0}}$ is depicted in fig. 14 along with unpolarised measurements. Keeping in mind that the large negative $\mathrm{CMR}^{\pi^{0}}$ of ref. [26] (full triangle tip down) is practically excluded by the $S_{0+}$ results discussed in sect. 3.2.1, very convincing agreement is observed between the various measurements. A recent very high statistics measurement of the angular distributions of recoil polarisation [43] found, just outside the range of fig. 14, $\mathrm{CMR}^{\pi^{0}}=(-6.61 \pm 0.18) \%$ at $Q^{2}=1 \mathrm{GeV} / \mathrm{c}^{2}$. This agreement extends also to the positive $\Im S_{0+} / M_{1+}$ ratio determined from $\rho_{L T}$.

The three polarisation components measured in parallel kinematics are model independently related by angular

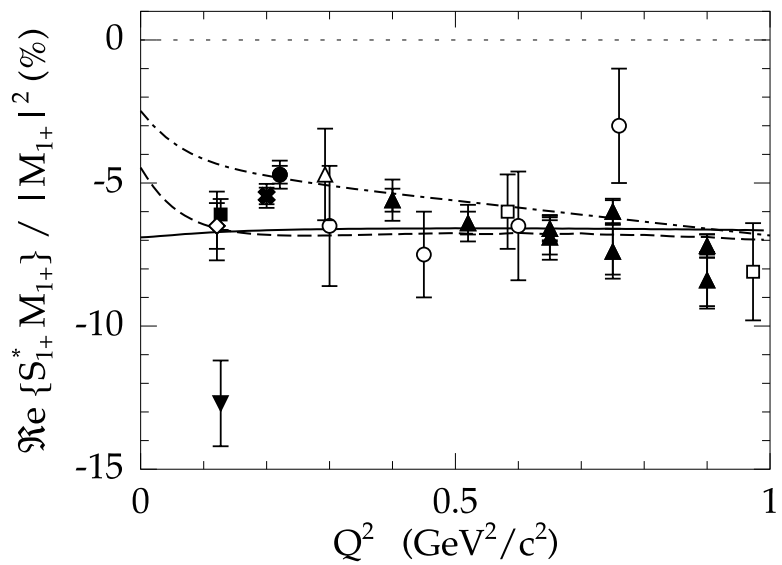

Fig. 14. $\mathrm{CMR}^{\pi^{0}}$ from recoil polarisation (open diamond) [38] in comparison to unpolarised measurements $[18,19,26,28$, $29,44,45]$ at low $Q^{2}$. The curves show model calculations MAID2003 [23] (solid), DMT2001 [25] (dashed) and Sato/Lee [22] (dashed dotted).

momentum conservation ${ }^{1}[46]$ :

$$
\chi_{x}^{2}+\chi_{y}^{2}=\frac{1}{\epsilon_{L}} \chi_{z}\left(1-\chi_{z}\right) .
$$

The reduced polarisations are defined by

$$
\begin{aligned}
& \chi_{x}=\frac{1}{P_{e} c_{-}} \cdot P_{x}=\frac{R_{L T^{\prime}}^{t}}{R_{T}+\epsilon_{L} R_{L}}, \\
& \chi_{y}=\frac{1}{c_{+}} \cdot P_{y}=\frac{R_{L T}^{n}}{R_{T}+\epsilon_{L} R_{L}}, \\
& \chi_{z}=\frac{1}{P_{e} c_{0}} \cdot P_{z}=\frac{R_{T T^{\prime}}^{l}}{R_{T}+\epsilon_{L} R_{L}} .
\end{aligned}
$$

The consistency relation seems hardly fulfilled by the measured recoil polarisations. The experimental result for eq. (33) is

$$
3.9 \pm 0.4_{\text {stat }} \pm 0.4_{\text {syst }}=7.9 \pm 0.7_{\text {stat }} \pm 1.2_{\text {syst }} .
$$

Despite the non-linear error propagation on the r.h.s. of eq. (33), the probability for such a finding is only a few percent. While MAID2000 fulfills the consistency relation, there is a discrepancy with the $P_{y}$ measurement. The MAID-refit mentioned above slightly improves this situation as shown in fig. 15, where the MAMI recoil polarisation data [38] are compared to the MAID versions 2000, 2003 and the re-fit. Nevertheless, the role of nonresonant background, which particularly shows up in $P_{y}$, still remains unresolved. Another measurement of $P_{y}$ [48] is in agreement with the MAMI data but has a large error. Unfortunately, ref. [43] gives no explicit values of the measured polarisations.

\footnotetext{
1 Despite the occurence of $\epsilon_{L}$ in eq. (33), the polarisation relation is nevertheless frame independent. The longitudinal polarisation parameter is also contained in the definition of the factors $c_{ \pm}$of the reduced polarisations and thus could be eliminated. A similar relation holds in elastic electron nucleon scattering, where $\chi_{y}$ vanishes [47].
} 

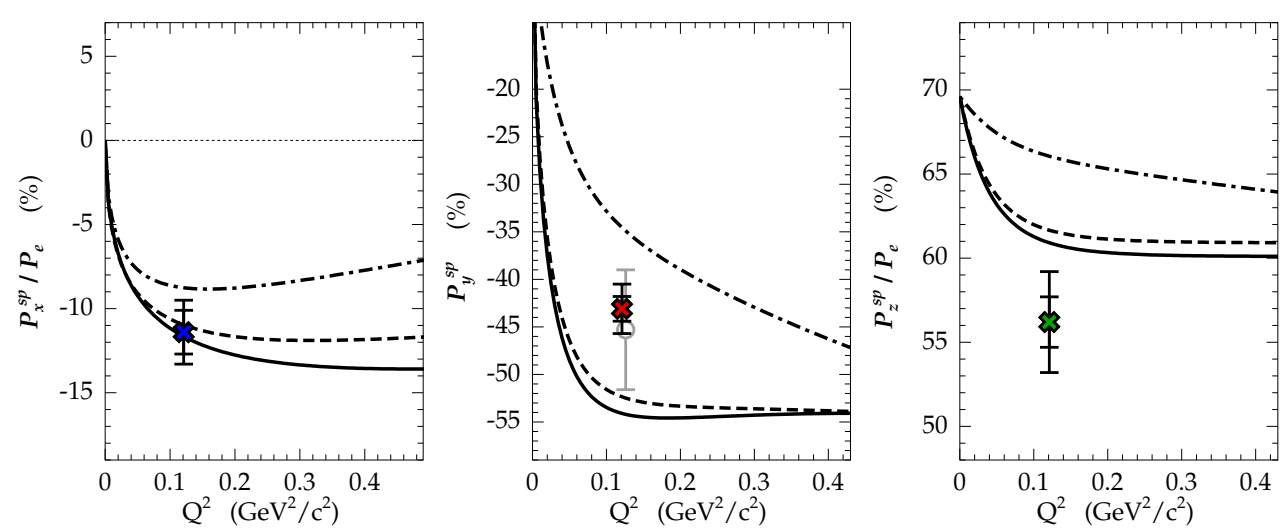

Fig. 15. Components of recoil polarisation measured at the energy of the $\Delta(1232)$-resonance in the reaction $p\left(\boldsymbol{e}, e^{\prime} \boldsymbol{p}\right) \pi^{0}[38]$. Curves show the MAID versions 2000 (full), 2003 (dashed), and the re-fit (dashed-dotted) on basis of $\rho_{L T}$ and $\rho_{L T^{\prime}}$ [21] discussed in the text. Note the suppressed zero middle and right.

In principle, from the reduced polarisations it is possible to determine the ratio of longitudinal to transverse response, $R_{L} / R_{T}$, without the need of a classical Rosenbluth separation. According to ref. [46] this can be achieved in three different ways, using either

- only the longitudinal component, $\chi_{z}$ (cf. also [49]),

- the quadratic sum of the transverse components, $\chi_{x}^{2}+\chi_{y}^{2}$, or

- all three reduced polarisations.

However, as a reflection of the almost violated consistency relation the results obtained from the MAMI measurements vary significantly. The smallest value, $R_{L} / R_{T}=$ $\left(4.7 \pm 0.4_{\text {stat }} \pm 0.6_{\text {syst }}\right) \%$, is extracted from the quadratic sum $\chi_{x}^{2}+\chi_{y}^{2}$ of the transverse reduced polarisations and the largest one, $R_{L} / R_{T}=\left(12.2_{-1.6_{\text {stat }}}^{+1.7}{ }_{-2.7}^{+2.9}\right.$ syst $) \%$, from $\chi_{z}$ alone. This presently prohibits a reliable extraction of $R_{L} / R_{T}$ but stresses the importance of a simultaneous measurement of all polarisation components with further improved accuracy.

\section{Interpretation}

The MAMI experiments towards the quadrupole strength in the $N \rightarrow \Delta(1232)$ transition yield very consistent results. The first order physical background contributions like $\Im m S_{0+}$ are now much better under control. Remaining uncertainties and inconsistencies seem related to the contribution of higher partial waves especially in the imaginary parts of interferences as in $\rho_{L T^{\prime}}$ and $P_{y}$. Though important for our understanding of the non-resonant processes, they are very much suppressed in the discussed observables with high sensitivity to EMR and CMR. At least in the vicinity of the photon point, a reliable extraction of the EMR and CMR is possible with a remaining relative model uncertainty of the order $\leq 10 \%$. Thus it is evident that the experimental results are an order of magnitude larger than expected from the quark model calculations.
Obviously, pionic degrees of freedom - which in a microscopic picture would be responsible for $e . g$. the $\Im m S_{0+}$ contribution (fig. 10) - play an important role. This is made particularly transparent within the dynamical models $[24,22]$. Such calculations can be split into the so-called bare and dressed parts, which represent the nucleon/delta core, and the pion cloud, respectively. This is also attempted within dispersion relation approaches [50]. While, in general, such a separation suffers from an unitary ambiguity [51], the models yield consistent results. E.g., the full model of ref. [24] describes the data while the bare calculation yields a very small and positive CMR. Moreover, the full calculation yields an $M_{1+}^{3 / 2}$ amplitude in agreement with the experimental data. In contrast, the bare calculation gives only about two thirds of $M_{1+}^{3 / 2}$ and a CMR which is an order of magnitude too small. This is similar to quark model calculations without pion degrees of freedom $[52,53]$, which also underestimate the quadrupole strength by a factor of ten and get only about $60 \%$ of the experimental $M_{1+}$.

Despite the yet unsatisfactory statistics of dynamic lattice calculations [54], those are in qualitative agreement with the experimental results and hence further support the important role of the pion cloud.

As outlined in the introduction the quadrupole transition strength can be interpreted in terms of a deformation of the baryons involved. Buchmann and Henley find opposite deformations of equal strength for nucleon and $\Delta$ [55]. Also the quadrupole moments for the nucleon core and the pion cloud are of opposite sign. However, the core appears almost spherical and the deformation due to the pion cloud is an order of magnitude stronger, very similar to the observation within the dynamical model. Buchmann and Henley quote total quadrupole moments in the range of $Q_{\text {nucl }}= \pm(0.113 \ldots 0.5) \mathrm{fm}^{2}$ for nucleon (upper sign) and $\Delta$ (lower sign). It is interesting to note that, relative to the size of the objects, the corresponding deformation

$$
\frac{Q_{\text {nucl }}}{\pi r_{N}^{2}}>0.043 \quad \text { of the nucleon }
$$


compared to

$$
\frac{Q_{\text {deut }}}{\pi r_{d}^{2}}=0.07 \quad \text { of the deuteron }
$$

would be of similar magnitude.

\section{Summary and outlook}

The MAMI experiments on pion photo- and electroproduction provided cornerstones for our understanding of the $N \rightarrow \Delta(1232)$ transition. At the photon point and at low $Q^{2}$ they unambiguously demonstrated that the quadrupole strength is an order of magnitude larger than expected in QCD-motivated constituent quark models.

Often a precursor for similar experiments at other laboratories, polarisation techniques played a key role. They have already been extended to polarised target measurements and to energies slightly above the $\Delta(1232)$ [56]. In view of the higher resonances which become accessible with the increased energy of MAMI C, their potential seems not at all exhausted yet. Of particular interest will be the determination of $Q^{2}$-slopes of transition amplitudes to Roper, $N(1440) P_{11}$, and $N(1535) S_{11}$ resonances. Since related to the spatial extention of the systems, new insights into the debated hybrid or molecular structure of such states can be expected. Extension of the experimental techniques to associated strangeness production seems relatively straightforward, provided that Kaons can be unambiguously identified and sufficiently high beam energy is available.

In particular through polarisation data of unprecedented accuracy MAMI has the potential to continuously challenge our understanding of the many-body structure of the nucleon. With all the virtues of the electromagnetic probe it can provide experimental key observables also for the comparison to the progressivly improving Lattice calculations.

It is a special pleasure for me to thank the retirees Hartmuth Arenhövel, Hartmut Backe, Dieter Drechsel, Jörg Friedrich, Karl-Heinz Kaiser and Thomas Walcher who, through their invaluable individual contributions over the last two decades, made it possible to turn MAMI into the big success it has become. I also greatfully acknowledge the support of D. Elsner in preparing talk and written manuscript of this overview.

\section{References}

1. F. Wilczek, hep-ph/0201222, MIT-CTP-3236.

2. A. Thomas, W. Weise, The Structure of the Nucleon (Wiley-VCH 2001).

3. S. Aoki et al., Phys. Rev. Lett. 84, 238 (2000).

4. Q. Mason et al., Phys. Rev. Lett. 95, 052002 (2005).

5. J.W. Negele, hep-lat/0509101 and references therein.

6. A. de Rújula, H. Georgi, S.L. Glashow, Phys. Rev. D 12, 147 (1975).

7. S.L. Glashow, Physica A 96, 27 (1979).

8. Compilation by J. Ahrens, Mainz (2000).

9. M. MacCormick et al., Phys. Rev. C 53, 41 (1996).

10. T.A. Armstrong et al., Phys. Rev. D 5, 1640 (1972).
11. D. Babusci et al., Phys. Rev. C 57, 291 (1998).

12. D. Drechsel, L. Tiator, J. Phys. G: Nucl. Part. Phys. 18, 449 (1992).

13. G. Knöchlein, D. Drechsel, L. Tiator, Z. Phys. A 352, 327 (1995).

14. R. Beck et al., Phys. Rev. Lett. 78, 606 (1997); 79, 4515 (1997) (E).

15. R. Beck et al., Phys. Rev. C 61, 035204 (2000).

16. O. Hanstein, D. Drechsel, L. Tiator, Nucl. Phys. A 632, 561 (1998).

17. R. Leukel, doctoral thesis, Mainz (2001).

18. K. Joo et al., Phys. Rev. Lett. 88, 122001 (2002).

19. R.W. Gothe, Prog. Part. Nucl. Phys. 44, 185 (2000).

20. V.V. Frolov et al., Phys. Rev. Lett. 82, 45 (1999).

21. D. Elsner et al., Eur. Phys. J. A 27, 91 (2006).

22. T. Sato, T.-S.H. Lee, Phys. Rev. C 63, 055201 (2001).

23. D. Drechsel, O. Hanstein, S.S. Kamalov, L. Tiator, Nucl. Phys. A 645, 145 (1999); http: //www.kph.uni-mainz.de/ MAID/.

24. S.S. Kamalov, S.N. Yang, Phys. Rev. Lett. 83, 4494 (1999).

25. S.S. Kamalov, S.N. Yang, D. Drechsel, L. Tiator, Phys. Rev. C 64, 032201 (2001).

26. F. Kalleicher et al., Z. Phys. A 359, 201 (1997).

27. H. Schmieden, Proceedings of NSTAR2001, edited by D. Drechsel, L. Tiator (World Scientific, 2001) p. 27.

28. J.C. Alder et al., Nucl. Phys. B 46, 573 (1972).

29. R. Siddle et al., Nucl. Phys. B 35, 93 (1971).

30. K. Aulenbacher, these proceedings.

31. L.G. Levchuk, Nucl. Instrum. Methods A 345, 496 (1994).

32. P. Bartsch, diploma thesis KPH 11/96, Mainz (1996).

33. K.I. Blomqvist et al., Nucl. Instrum. Methods A 403, 263 (1998).

34. P. Bartsch et al., Phys. Rev. Lett. 88, 142001 (2002).

35. K.M. Watson, Phys. Rev. 95, 228 (1954).

36. E. Fermi, Suppl. Nuovo Cimento 2, 17 (1955).

37. H. Schmieden, Eur. Phys. J. A 1, 427 (1998).

38. Th. Pospischil et al., Phys. Rev. Lett. 86, 2959 (2001).

39. Th. Pospischil et al., Nucl. Instrum. Methods A 483, 713 (2002).

40. Th. Pospischil et al., Nucl. Instrum. Methods A 483, 726 (2002).

41. E. Aprile-Giboni et al., Nucl. Instrum. Methods 215, 147 (1983).

42. M.W. McNaughton et al., Nucl. Instrum. Methods 241, 435 (1985).

43. J.J. Kelly et al., Phys. Rev. Lett. 95, 102001 (2005); nuclex/0509004.

44. K. Bätzner et al., Nucl. Phys. B 76, 1 (1974).

45. N.F. Sparveris et al., Phys. Rev. Lett. 94, 022003 (2005).

46. H. Schmieden, L. Tiator, Eur. Phys. J. A 8, 15 (2000).

47. Th. Pospischil et al., Eur. Phys. J. A 12, 125 (2001).

48. G.A. Warren et al., Phys. Rev. C 58, 3722 (1998).

49. J.J. Kelly, Phys. Rev. C 60, 054611 (1999).

50. I.G. Aznauryan, S.G. Stepanyan, Phys. Rev. D 59, 054009 (1999).

51. P. Wilhelm et al., Phys. Rev. C 54, 1423 (1996).

52. N. Isgur, G. Karl, R. Koniuk, Phys. Rev. D 25, 2394 (1982).

53. S.S. Gershtein, G.V. Dzhikiya, Sov. J. Nucl. Phys. 34, 870 (1981).

54. C. Alexandrou et al., Phys. Rev. Lett. 94, 021601 (2005); hep-lat/0509140.

55. A.J. Buchmann, E.M. Henley, Phys. Rev. C 63, 015202 (2000).

56. A. Thomas, these proceedings. 